
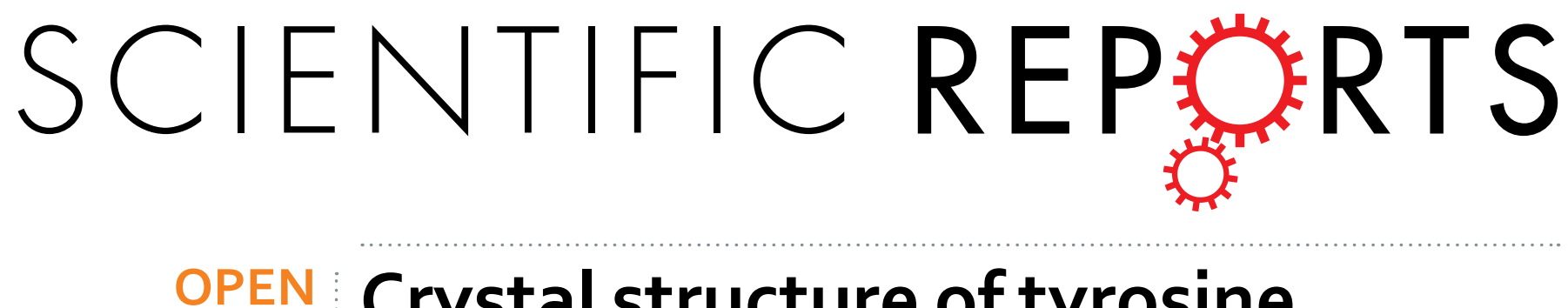

\title{
Crystal structure of tyrosine decarboxylase and identification of key residues involved in
}

Received: 09 February 2016

Accepted: 23 May 2016

Published: 13 June 2016

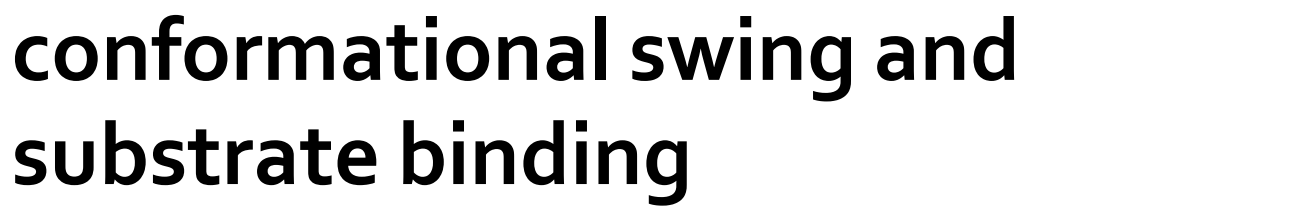

Haixia Zhu ${ }^{1,2}$, Guochao Xu ${ }^{1}$, Kai Zhang ${ }^{1}$, Xudong Kong ${ }^{3}$, Ruizhi Han ${ }^{1}$, Jiahai Zhou ${ }^{2}$ \&e Ni ${ }^{1}$

Tyrosine decarboxylase (TDC) is a pyridoxal 5-phosphate (PLP)-dependent enzyme and is mainly responsible for the synthesis of tyramine, an important biogenic amine. In this study, the crystal structures of the apo and holo forms of Lactobacillus brevis TDC ( $L b T D C)$ were determined. The LbTDC displays only $25 \%$ sequence identity with the only reported TDC structure. Site-directed mutagenesis of the conformationally flexible sites and catalytic center was performed to investigate the potential catalytic mechanism. It was found that $\mathrm{H} 241$ in the active site plays an important role in PLP binding because it has different conformations in the apo and holo structures of $\angle b T D C$. After binding to PLP, $\mathrm{H} 241$ rotated to the position adjacent to the PLP pyridine ring. Alanine scanning mutagenesis revealed several crucial regions that determine the substrate specificity and catalytic activity. Among the mutants, the S586A variant displayed increased catalytic efficiency and substrate affinity, which is attributed to decreased steric hindrance and increased hydrophobicity, as verified by the saturation mutagenesis at $\mathrm{S} 586$. Our results provide structural information about the residues important for the protein engineering of TDC to improve catalytic efficiency in the green manufacturing of tyramine.

Biogenic amines (BAs) are essential, biologically important nitrogenous compounds that play important roles in intercellular communication as major transmitters ${ }^{1}$. Many BAs, including dopamine, serotonin, histamine and tyramine, generally exist in microbial, plant and animal cells ${ }^{2}$. Tyramine is a naturally occurring BA and has diverse applications. It could act as an endogenous ligand for mammalian trace amine-associated receptors and as a biochemical precursor for dopamine and octopamine, which are neurotransmitters and neuromodulators in invertebrate nervous systems $\mathrm{s}^{3-6}$. Tyramine has also been shown to be an effective therapeutic agent in Diabetes Mellitus type II by functioning as an insulin mimic to stimulate glucose transport ${ }^{7}$, and as a key intermediate of Bezafibrate for the treatment of hyperlipidemia by reducing cholesterol and triglyceride levels ${ }^{8}$. Similar to dopamine, the presence of higher concentrations of tyramine in vivo could be regarded as an indicator of health problems, such as cardiovascular arrest/stroke. Although the industrial production of tyramine has been limited to chemical processes, enzymatic decarboxylation of tyrosine by a decarboxylase is much more promising, due to its green properties and high efficiency.

Amino acid decarboxylases are PLP-dependent enzymes that are responsible for the biosynthesis of BAs and polyamines, and can be divided into four evolutionally distant subgroups based on analyses of the primary sequence alignments and the conserved domains ${ }^{9}$. Tyrosine decarboxylase (TDC, EC 4.1.1.25) is classified into Group II and shares high identity with L-3,4-dihydroxy-L-phenylalanine (DOPA) decarboxylase (DDC), glutamic acid decarboxylase (GAD), and histidine decarboxylase (HDC). TDC could catalyze the decarboxylation

${ }^{1}$ The Key Laboratory of Industrial Biotechnology, Ministry of Education, School of Biotechnology, Jiangnan University, Wuxi 214122, Jiangsu, People's Republic of China. ${ }^{2}$ Shanghai Institute of Organic Chemistry, State Key Laboratory of Bioorganic \& Natural Products Chemistry, Chinese Academy of Sciences, Shanghai 200032, People's Republic of China. ${ }^{3}$ State Key Laboratory of Bioreactor Engineering, School of Biotechnology, East China University of Science and Technology, Shanghai 200237, People's Republic of China. Correspondence and requests for materials should be addressed to J.Z. (email: jiahai@mail.sioc.ac.cn) orY.N. (email: yni@jiangnan.edu.cn) 


\begin{tabular}{|c|c|c|}
\hline & 5HSI (without PLP) & 5HSJ (complex with PLP) \\
\hline \multicolumn{3}{|l|}{ Data collection statistics } \\
\hline \multicolumn{3}{|l|}{ Cell parameters } \\
\hline a, b, c $(\AA)$ & $61.8,126.2,84.8$ & $61.9,126.8,82.9$ \\
\hline$\alpha, \beta, \gamma\left(^{\circ}\right)$ & $90.0,113.4,90.0$ & $90.0,109.7,90.0$ \\
\hline Resolution $(\AA)$ & $50-1.73(1.79-1.73)$ & $50-1.90(1.97-1.90)$ \\
\hline Wavelength $(\AA)$ & 1.54178 & 0.97908 \\
\hline Space group & $\mathrm{P} 2_{1}$ & $\mathrm{P} 2_{1}$ \\
\hline Total reflections & 483537 & 488739 \\
\hline Completeness (\%) & $90.7(89.2)$ & $99.7(99.9)$ \\
\hline $\mathrm{R}_{\text {merge }}(\%)^{\mathrm{b}}$ & $5.1(23.8)$ & $11.9(77.0)$ \\
\hline Redundancy & $4.3(4.3)$ & $5.2(5.2)$ \\
\hline I/error & $12.4(3.8)$ & $10.0(3.0)$ \\
\hline \multicolumn{3}{|l|}{ Refinement statistics } \\
\hline $\mathrm{R}_{\text {work }} / \mathrm{R}_{\text {free }}(\%)$ & $15.52 / 19.05$ & $17.13 / 21.28$ \\
\hline \multicolumn{3}{|l|}{ Average B factor $\left(\AA^{2}\right)$} \\
\hline Protein & 25.1 & 33.1 \\
\hline Ligand & 23.8 & 32.2 \\
\hline Solvent & 34.3 & 37.2 \\
\hline \multicolumn{3}{|c|}{ R.M.S. deviations from ideal geometry } \\
\hline Bond length $(\AA)$ & 0.006 & 0.007 \\
\hline Bond angle $\left({ }^{\circ}\right)$ & 1.074 & 1.041 \\
\hline \multicolumn{3}{|l|}{ Ramachandran plot } \\
\hline Favored (\%) & 97.06 & 97.65 \\
\hline Allowed (\%) & 2.61 & 2.10 \\
\hline Outliers (\%) & 0.34 & 0.25 \\
\hline \multicolumn{3}{|l|}{ No. atoms } \\
\hline No. of non-hydrogen atoms & 10,743 & 10,144 \\
\hline No. of amino acid residues & 9,541 & 9,542 \\
\hline No. of solvent molecules & 1,201 & 569 \\
\hline
\end{tabular}

Table 1. Data collection and refinement statistics of the TDC structure alone and in complex with PLP. ${ }^{a}$ The values in parentheses represent the highest resolution shell. ${ }^{\mathrm{b}} \mathrm{R}_{\text {merge }}=\sum_{h k l}\left|I_{i}-I_{m}\right| / I_{h k l} I_{m}$, where $I_{\mathrm{i}}$ and $I_{\mathrm{m}}$ are the observed intensity and the mean intensity of related reflections, respectively. The values in parentheses indicate the highest resolution shell.

of L-tyrosine and L-DOPA to tyramine and dopamine ${ }^{10,11}$. To date, various TDCs have been identified from different organisms, such as plants ${ }^{12,13}$, insects ${ }^{14,15}$, and different microorganisms including Lactobacillus bre$v_{i s}^{10,16}$, Enterococcus faecalis ${ }^{17}$, Methanocaldococcus jannaschii ${ }^{18}$, Sporolactobacillus sp. ${ }^{19}$, and Lactococcus lactis ${ }^{20}$. Previous studies show that lactic acid bacteria (LAB) are one major class of TDC producers. From an evolutionary point of view, the TDC system in LAB is responsible for tyramine formation in response to acid challenge. The TDC system could generate a proton motive force (PMF) through proton consumption in the decarboxylation reaction and the membrane potential resulting from electrogenic transport of tyrosine in exchange for its corresponding biogenic amine tyramine ${ }^{21}$. However, despite its important applications, only one TDC structure from Methanococcus jannaschii (PDB code: 3F9T, MjTDC) has been resolved and deposited in the PDB database, and the key residues responsible for substrate binding and the catalytic mechanism remain unclear.

In our previous study, we identified a TDC coding gene from Lactobacillus brevis CGMCC 1.2028 and successfully expressed the soluble protein in Escherichia coli BL21(DE3) ${ }^{10}$. A BLASTp search based on the amino acid sequence of Lactobacillus brevis TDC ( $L b T D C$ ) reveals that $L b$ TDC shares only $25 \%$ sequence identity and $60 \%$ coverage with $M j$ TDC. Importantly, $L b$ TDC is superior to $M j T D C$ due to its lower $K_{\mathrm{m}}(0.58$ versus $1.6 \mathrm{mM}$ ) and higher specific activity (43.1 versus $\left.1.1 \mathrm{U} \cdot \mathrm{mg}^{-1}\right)^{18}$. To better understand its catalytic mechanism, the $L b T D C$ protein was crystallized and the X-ray diffraction patterns were collected in this study. Furthermore, structure-guided site-directed mutagenesis and alanine scanning and saturation mutagenesis of $L b T D C$ were performed on residues around the substrate binding sites and those required for conformational stability to elucidate the function of key residues involved in the catalytic mechanism and to promote the potential applications of $L b$ TDC in tyramine synthesis, food safety, and pharmacology.

\section{Materials and Methods}

Chemicals, reagents, strains and plasmids. Tyrosine, PLP, tyramine, DOPA, dopamine, SeMet and all reagents for protein crystallization were purchased from Sigma-Aldrich Co., Ltd. (St. Louis, MO, USA). E. coli BL21(DE3) harboring pET24a-tdc was constructed in our previous work ${ }^{10}$. Phanta HS Super-Fidelity DNA Polymerase was obtained from Vazyme Biotech Co., Ltd. (Nanjing, China), and DpnI was obtained from Toyobo Co., Ltd. (Osaka, Japan). 


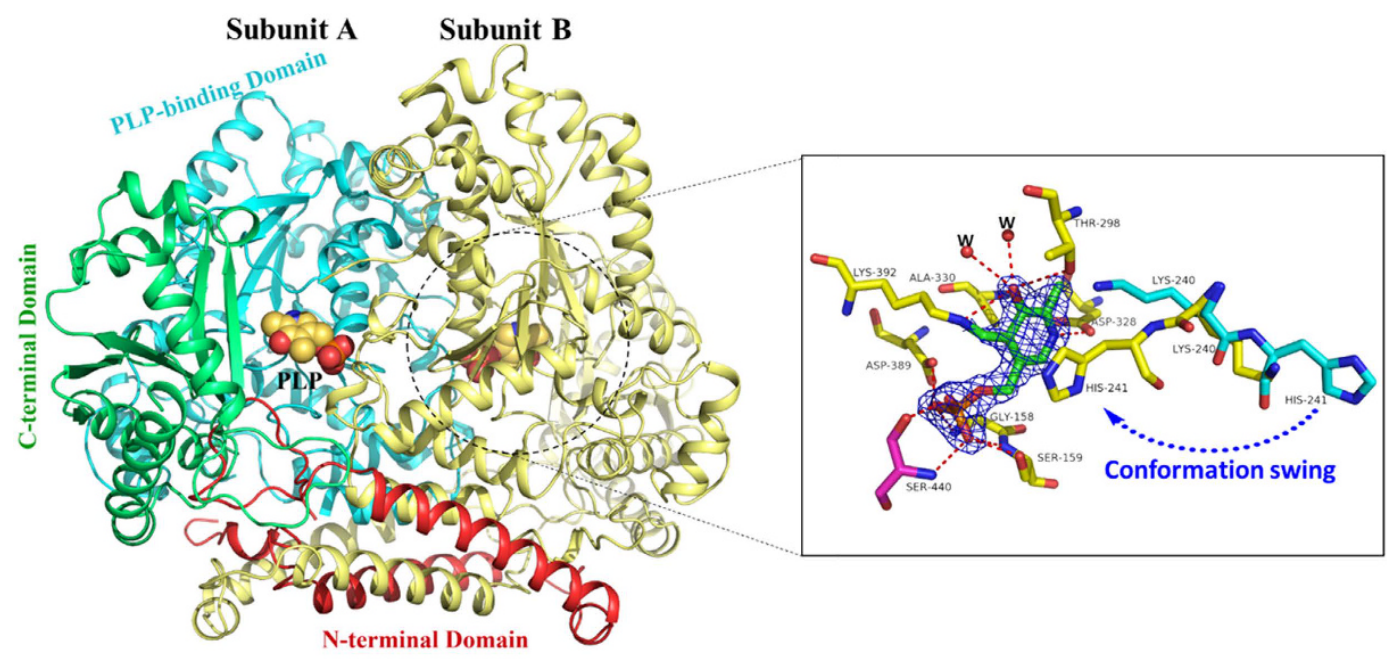

Figure 1. Overall crystal structure of $\boldsymbol{L} b$ TDC in complex with PLP. The $N$-terminal domain (red), PLPbinding (cyan) and C-terminal (green) domains of subunit A are labeled, and subunit B is colored in yellow. PLP is shown as spheres. The different conformations of K240 and H241 in apo-LbTDC and holo- $L b$ TDC are shown in the box. Residue S440 from the other subunit is shown in magenta. The 2Fo-Fc map omitting PLP contoured at $2 \sigma$ is shown (mesh). Hydrogen bonds are depicted as red dashed lines. Water molecules are shown as red spheres.

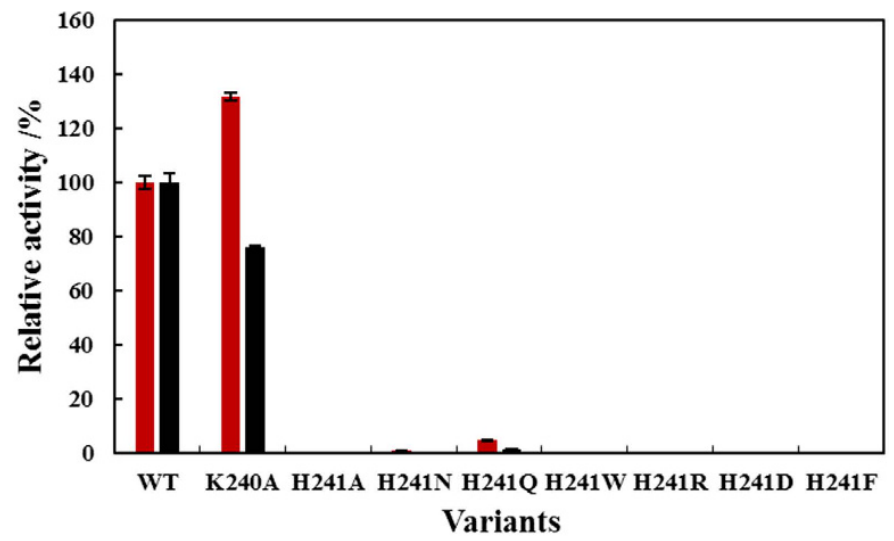

Figure 2. Mutagenesis of K240 and H241. ( $\square$ ) tyrosine, ( $\square$ ) DOPA. The activities of the WT enzyme toward tyrosine and DOPA are set to $100 \%$.

Protein expression and purification. Recombinant E. coli BL21(DE3) harboring pET24a- $t d c$ was cultivated at $37^{\circ} \mathrm{C}$ and $180 \mathrm{rpm}$ in a LB medium supplemented with $1 \%$ glucose and $50 \mu \mathrm{g} \mathrm{mL}^{-1}$ kanamycin until the $\mathrm{OD}_{600}$ reached $0.6-0.8$; then, $0.2 \mathrm{mM}$ isopropyl- $\beta$-D-thiogalactopyraniside (IPTG) was added, and the bacteria were further cultured at $16^{\circ} \mathrm{C}$. After induction for $16 \mathrm{~h}$, the cells were harvested by centrifugation $(8,000 \times g$ for $10 \mathrm{~min})$, resuspended in buffer A $(25 \mathrm{mM}$ Tris- $\mathrm{HCl}, 300 \mathrm{mM} \mathrm{NaCl}, 20 \mathrm{mM}$ imidazole, $5 \mathrm{mM} \beta$-mercaptoethanol, pH 7.4, and $1 \mathrm{mM}$ phenyl-methyl-sulfonyl fluoride), and then disrupted using a high-pressure homogenizer (ATS BASIC-II, Canada). The cell lysate was centrifuged $(15,000 \times g$ for $30 \mathrm{~min})$ at $4{ }^{\circ} \mathrm{C}$ to remove the cell debris, and the resulting supernatant was loaded onto a Fast Flow $\mathrm{Ni}^{2+}$-agarose column (GE Healthcare, USA), which was pre-equilibrated with buffer A. Afterwards, the retained protein was eluted with a $20-300 \mathrm{mM}$ imidazole gradient in buffer B (25 mM Tris- $\mathrm{HCl}, 300 \mathrm{mM} \mathrm{NaCl}, 300 \mathrm{mM}$ imidazole, and $5 \mathrm{mM} \beta$-mercaptoethanol, $\mathrm{pH}$ 7.4). The eluents were concentrated, desalted, and stored in buffer containing $25 \mathrm{mM}$ Tris- $\mathrm{HCl}, 150 \mathrm{mM} \mathrm{NaCl}$, and $1 \mathrm{mM}$ DL-Dithiothreitol, $\mathrm{pH}$ 7.4. After the eluents were concentrated to approximately $10 \mathrm{mg} \cdot \mathrm{mL}^{-1}$, the purified apo- $L b$ TDC protein was immediately frozen in liquid nitrogen and stored at $-80^{\circ} \mathrm{C}$ for crystallization. The molecular weight was analyzed by SDS-PAGE and Superdex-200 gel filtration (GE Healthcare, USA). The selenomethionine-substituted $L b \mathrm{TDC}$ (SeMet- $L b \mathrm{TDC}$ ) protein was prepared using previously reported methods $^{22}$ and purified as described above. 


\begin{tabular}{|c|c|c|c|c|c|}
\hline Substrate & Enzyme & $\begin{array}{c}\text { Specific activity } \\
{\left[\mathrm{U} \cdot \mathrm{mg}^{-1}\right]}\end{array}$ & $K_{\mathrm{m}}[\mathrm{mM}]$ & $k_{\text {cat }}\left[\mathrm{s}^{-1}\right]$ & $\begin{array}{c}k_{\mathrm{cat}} / K_{\mathrm{m}} \\
{\left[\mathrm{s}^{-1} \cdot \mathrm{mM}^{-1}\right]}\end{array}$ \\
\hline \multirow{7}{*}{ Tyrosine } & WT & $43.1 \pm 1.0$ & $0.6 \pm 0.1$ & $124.8 \pm 1.5$ & 216.0 \\
\hline & K240A & $56.8 \pm 0.7$ & $1.6 \pm 0.2$ & $224.5 \pm 11.9$ & 139.4 \\
\hline & $\mathrm{H} 241 \mathrm{~N}$ & $0.2 \pm 0.1$ & $\mathrm{ND}^{\mathrm{a}}$ & ND & ND \\
\hline & H241Q & $1.9 \pm 0.1$ & ND & ND & ND \\
\hline & S586A & $96.0 \pm 2.0$ & $0.4 \pm 0.1$ & $250.9 \pm 4.2$ & 600.4 \\
\hline & Y398A & $0.6 \pm 0.1$ & $3.0 \pm 0.5$ & $9.8 \pm 0.8$ & 3.4 \\
\hline & Y398F & $4.2 \pm 0.2$ & $2.4 \pm 0.5$ & $31.9 \pm 3.4$ & 13.5 \\
\hline \multirow{8}{*}{ DOPA } & & & & & \\
\hline & WT & $26.5 \pm 0.9$ & $0.8 \pm 0.1$ & $76.5 \pm 3.0$ & 101.1 \\
\hline & K240A & $20.1 \pm 0.2$ & $1.1 \pm 0.2$ & $64.7 \pm 3.0$ & 58.3 \\
\hline & $\mathrm{H} 241 \mathrm{~N}$ & n.d. ${ }^{b}$ & ND & ND & ND \\
\hline & H241Q & $0.3 \pm 0.1$ & ND & ND & ND \\
\hline & S586A & $43.7 \pm 1.5$ & $0.7 \pm 0.1$ & $126 \pm 3.5$ & 181.3 \\
\hline & Y398A & $0.8 \pm 0.2$ & $1.7 \pm 0.2$ & $2.5 \pm 0.2$ & 1.5 \\
\hline & Y398F & $3.2 \pm 0.4$ & $0.7 \pm 0.1$ & $12.3 \pm 0.3$ & 18.4 \\
\hline
\end{tabular}

Table 2. Kinetic parameters of $L b T D C$ and the mutant proteins toward tyrosine and DOPA. ${ }^{\text {NND, not }}$ determined. ${ }^{b}$ n. d.: no activity was detected.
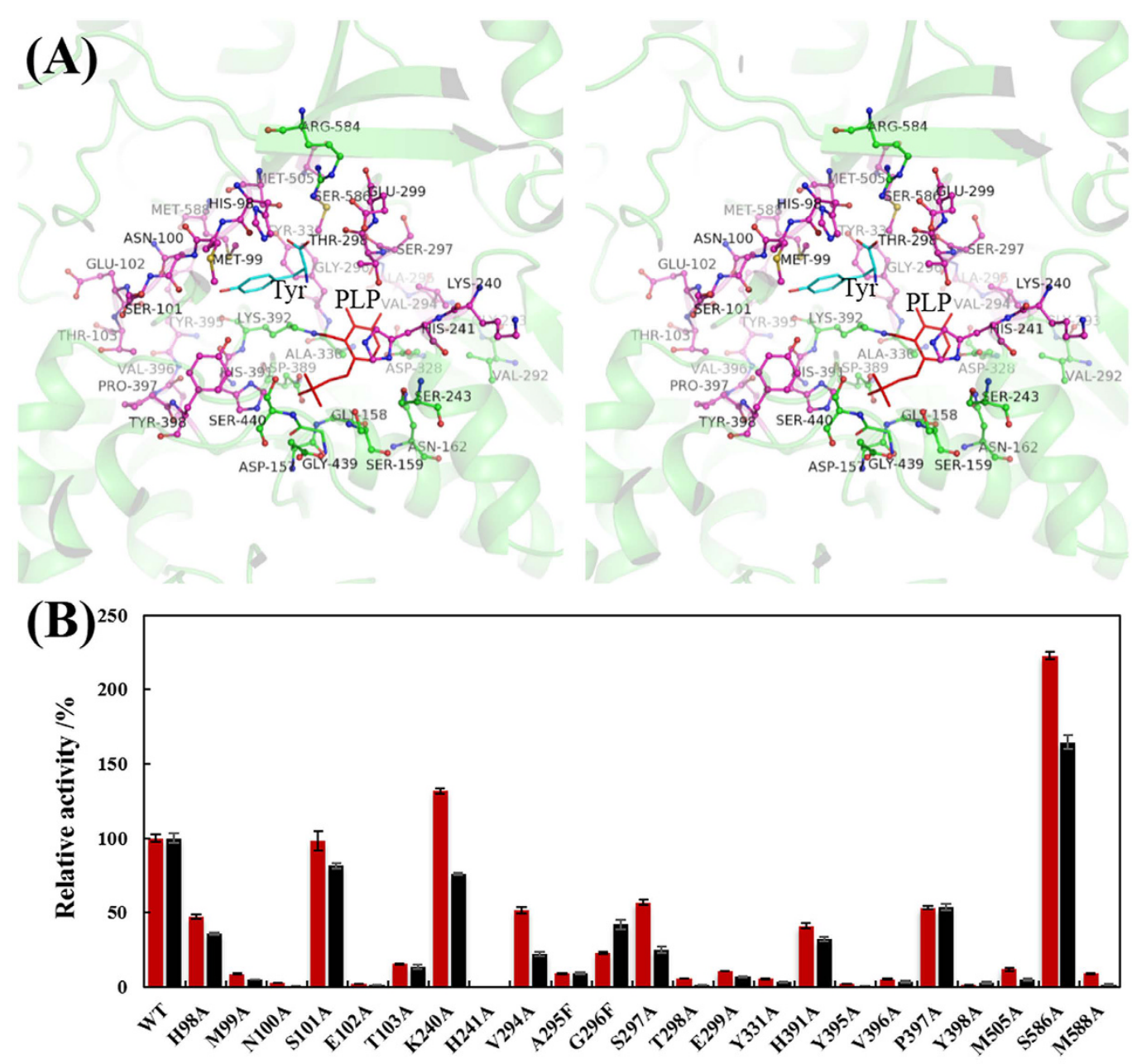

Variants

Figure 3. (A) Stereo views of the catalytic center of $L b T D C$. The residues that were mutated in this study are shown in magenta. (B) Relative activities of $L b T D C$ and its variants toward tyrosine and DOPA. PLP and the docked substrate tyrosine are shown in red and cyan, respectively. ( $\boldsymbol{\square}$ ) tyrosine, ( $\mathbf{\square})$ DOPA. The activities of the WT enzyme toward tyrosine and DOPA are set to $100 \%$. 


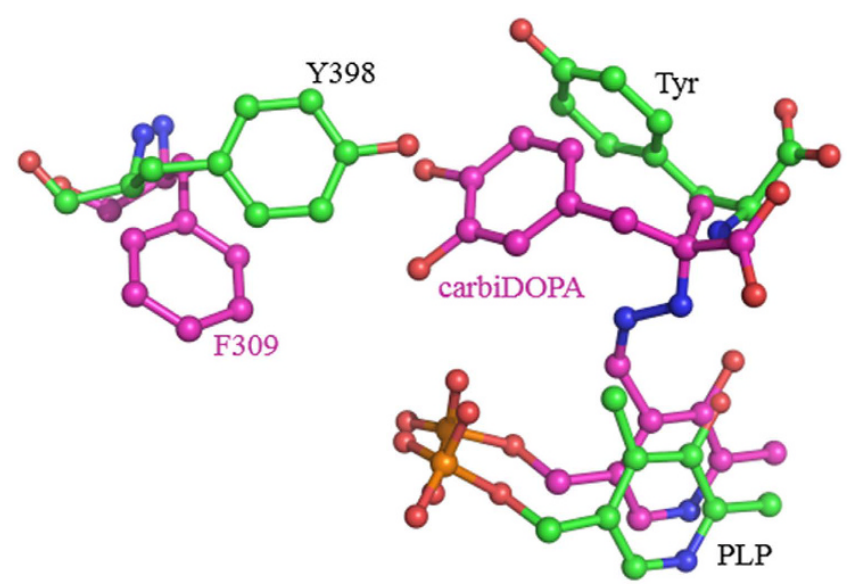

Figure 4. Superposition of the $L b$ TDC structure onto the pig DDC structure. Residues from pig DDC are colored in magenta, and those from $L b \mathrm{TDC}$ are colored in green.

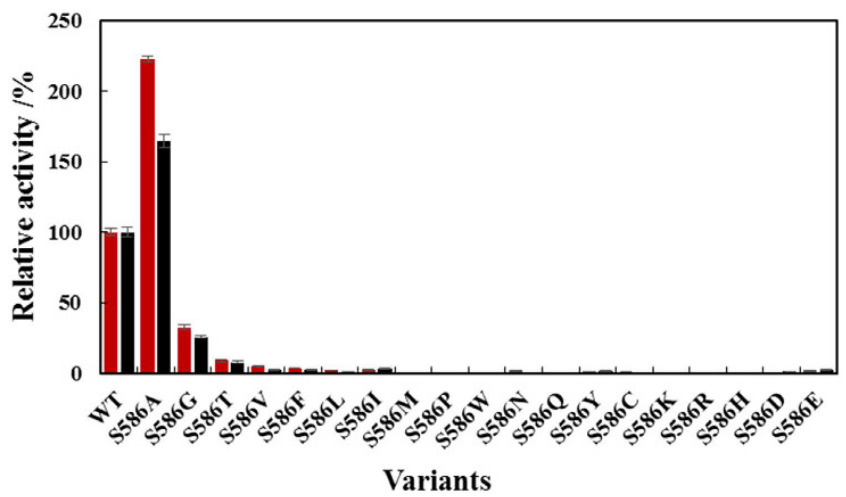

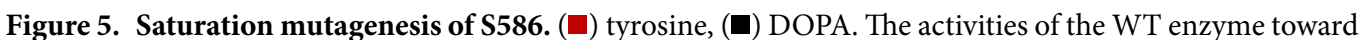
tyrosine and DOPA of the WT are set to $100 \%$.

Crystallization. Initial crystallization was performed at $18^{\circ} \mathrm{C}$ using the sitting-drop vapor diffusion method and commercial crystallization kits. Based on the obtained preliminary crystallization condition, the $\mathrm{pH}$ of the buffer and concentration of the precipitant were further optimized to obtain regular crystals. The best crystals were observed in the droplets obtained by mixing $2 \mu \mathrm{L}$ of protein solution $\left(10 \mathrm{mg} \cdot \mathrm{mL}^{-1}\right)$ with $2 \mu \mathrm{L}$ of reservoir solution, which consisted of $0.1 \mathrm{M}$ sodium cacodylate trihydrate ( $\mathrm{pH} 7.5), 0.2 \mathrm{M} \mathrm{MgCl}_{2}$, and $18 \%$ PEG 1000 . The SeMet-LbTDC crystals were obtained in $0.1 \mathrm{M}$ sodium cacodylate trihydrate, $\mathrm{pH} 7.0,0.1 \mathrm{M} \mathrm{MgCl}_{2}$, and $16 \%$ PEG 4000. The $L b T D C / P L P$ complex was also crystallized at molar ratio of 1:4 under the same conditions as SeMet-LbTDC. Crystals were usually obtained in 2 to 5 days.

Data collection, structure determination and refinement. For X-ray diffraction, all crystals were equilibrated in a cryoprotectant solution containing $90 \%$ reservoir solution and $10 \%$ glycerol and then flash-cooled with liquid nitrogen. Diffraction data for SeMet- $L b$ TDC were collected using beamline BL17A at the High Energy Accelerator Research Organization (KEK, Japan). The data for the native protein and $L b$ TDC-PLP complex were collected using beamlines BL17U1 and BL19U1 at the Shanghai Synchrotron Radiation Facility (SSRF, China), respectively. All of the diffraction images were integrated and scaled with the HKL2000 package ${ }^{23}$. The crystal parameters and data collection statistics are listed in Table 1.

The phases of $L b$ TDC were solved using the Multi-wavelength anomalous diffraction method. The position of the Se atom was identified by Autosol ${ }^{24}$. Then, AutoBuild ${ }^{25}$ in the Phenix package was employed to automatically build the model of $L b$ TDC. Several cycles of refinement were performed using Phenix ${ }^{26}$ and $\operatorname{Coot}^{27}$. The structure of the $L b$ TDC/PLP complex was solved by the molecular replacement method using Phaser $^{28}$ in the CCP 4 crystallographic suite ${ }^{29}$, and the crystal structure of LbTDC was used as a search model.

Mutagenesis. Site-directed mutagenesis of $L b$ TDC was performed by PCR using pET24a- $t d c$ as a template and Phanta HS Super-Fidelity DNA Polymerase. The resulting PCR products $(10 \mu \mathrm{L})$ were digested with $0.2 \mu \mathrm{L}$ of $D p n \mathrm{I}$ for $2 \mathrm{~h}$ at $37^{\circ} \mathrm{C}$ to remove the methylated templates. Then, the whole plasmids were transformed into $E$. 

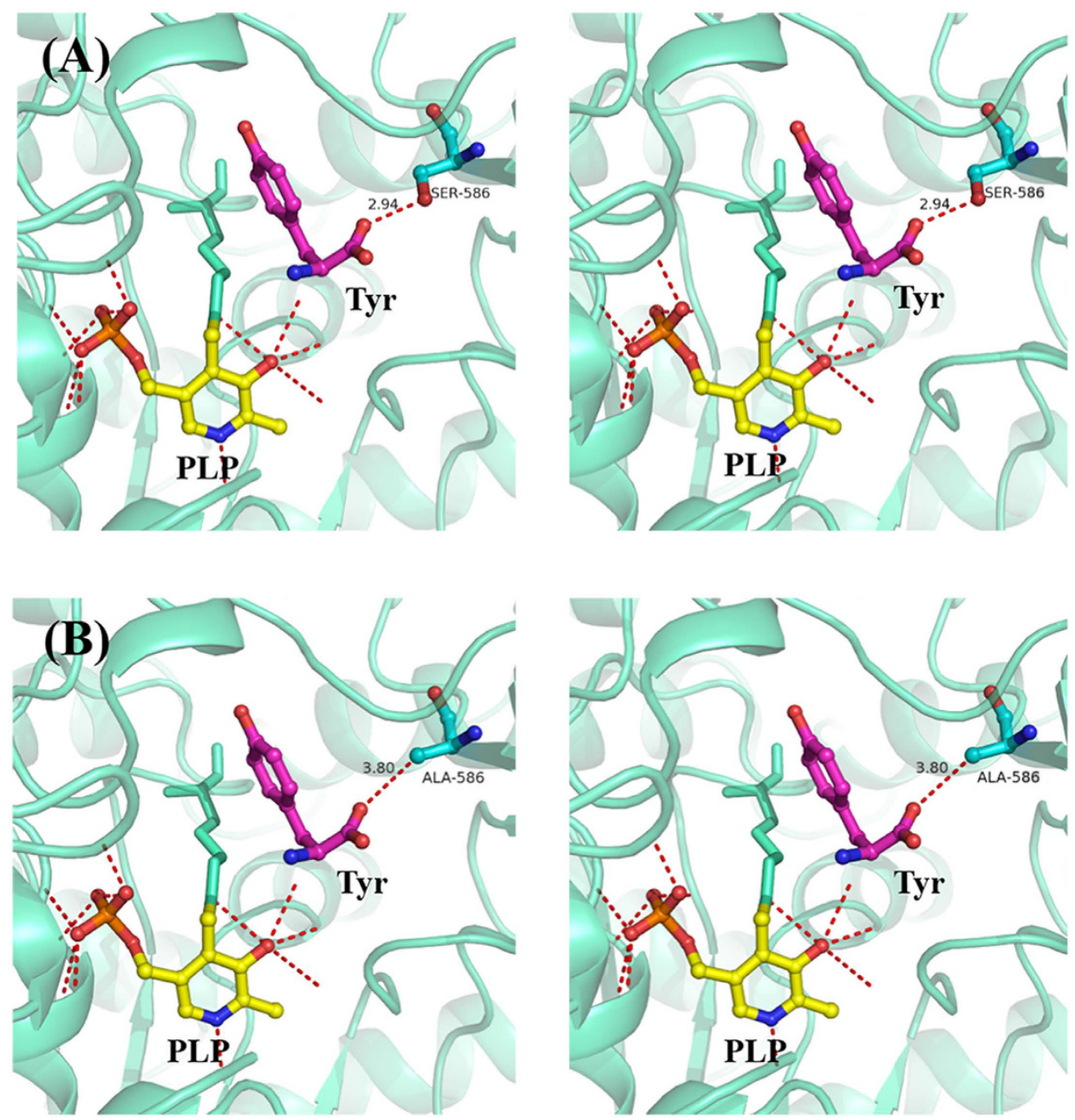

Figure 6. Stereo views of substrate binding of the WT and S586A. (A) S586 and (B) A586. PLP and the docked substrate tyrosine are shown in yellow and magenta, respectively. Residue interactions are depicted as red dashed lines.

coli JM109 and cultivated on LB agar plates containing $50 \mu \mathrm{g} \cdot \mathrm{mL}^{-1} \mathrm{kanamycin}$. The mutated plasmids were confirmed by sequencing and further transformed into E. coli BL21 (DE3) for over-expression. The primers used in this study are shown in Table S1.

Enzymatic activity and kinetic assay. The enzymatic activity of $L b T D C$ was determined using a previously reported method ${ }^{10}$, with some modifications. The assay mixture $(1 \mathrm{~mL})$ consisted of $2.75 \mathrm{mM}$ L-tyrosine or L-DOPA in sodium acetate buffer (0.2 M, pH 5.0), 0.2 mM PLP, and an appropriate amount of enzyme in buffer $\left(25 \mathrm{mM}\right.$ Tris- $\mathrm{HCl}$ and $150 \mathrm{mM} \mathrm{NaCl}, \mathrm{pH}$ 7.4). The reaction was incubated at $40^{\circ} \mathrm{C}$ for $10 \mathrm{~min}$ and terminated by boiling at $100^{\circ} \mathrm{C}$ for $10 \mathrm{~min}$. The tyrosine, tyramine, DOPA, and dopamine levels were quantified by HPLC (Agilent 1260, USA) equipped with a Diamonsil C18 column (DIKMA, China) using water/methanol $(90 / 10)$ as eluent at $1.0 \mathrm{~mL} \cdot \mathrm{min}^{-1}, 220 \mathrm{~nm}$, and $30^{\circ} \mathrm{C}$. One unit of activity was defined as the amount of enzyme that was required for the production of $1.0 \mu \mathrm{mol}$ tyramine or dopamine per minute under the above conditions. The kinetic parameters were determined in the presence of varying concentrations of tyrosine and DOPA from 0.1 to $5.5 \mathrm{mM}$ and calculated by non-linear curve fitting using Origin8.0. All assays were performed in triplicate.

Molecular docking. All docking calculations were accomplished with AutoDock Vina 1.1. A docking algorithm that takes into account the ligand flexibility but keeps the protein rigid was employed. Docking runs were performed using the standard parameters of the program for interactive growing and subsequent scoring. However, the parameters used to set the grid box dimensions and center were: center_x $=33.121$; center_y $=-7.691$; center_z $=77.977$; size_x $=15$; size_y $=15$; size_z $=15$.

\section{Results and Discussion}

Overall structures of $L \boldsymbol{b}$ TDC. After optimization of the crystallization conditions, the crystals of $L b T D C$ alone and in complex with PLP (apo- $L b T D C$ and holo- $L b T D C$, respectively) were obtained and the corresponding three-dimensional structures were resolved. The $L b T D C$ crystals were diffracted at $1.90 \AA$ and belonged to 
the space group $\mathrm{P} 2{ }_{1}$. The final structure was refined to $\mathrm{R}_{\text {work }}$ of $17.13 \%$ and $\mathrm{R}_{\text {free }}$ of $21.28 \%$. The crystallographic statistics for data collection and refinement are listed in Table 1. The structures of LbTDC alone and in complex with PLP have been deposited in the PDB database under accession Nos. 5HSI and 5HSJ.

The overall structure of $L b T D C$ is shown in Fig. $1 . L b T D C$ is a homodimer, and the two subunits exist in an asymmetric form. Compared with the open conformation of the apo form of the human DDC structure ${ }^{30}$, the structure of apo- $L b T D C$ is in a closed conformation. Because there are no cysteines in $L b T D C$, the two subunits are linked to each other by a hinge-like domain consisting of two long, parallel $\alpha$-helixes. Similar to the structures of other Group II decarboxylases, each $L b$ TDC monomer is composed of three distinct domains ${ }^{31}$ : the $N$-terminal (residues 7-105) domain, the PLP-binding (residues 106-462) domain, and the small (residues 463-618) domain. The $\mathrm{N}$-terminal domain is the hinge-like domain that intertwines between the two subunits and contributes to the stability of the dimer structure. This $N$-terminal domain is connected to the PLP-binding domain by a long loop. The PLP-binding domain is composed of a typical seven stranded $\beta$-sheet surrounded by thirteen $\alpha$-helixes, which form the dimer interface. The small (or C-terminal) domain contains a five-stranded $\beta$-sheet and five $\alpha$-helixes. The active center is located in a shallow cavity at the interface between the two subunits of the dimer. Residues from both subunits are involved in cofactor binding ${ }^{31}$.

Tyrosine decarboxylase from Methanococcus jannaschii (MjTDC) is the only TDC structure deposited in PDB under accession number 3F9T and was solved at 2.1 Å resolution. A comparison of the sequences of $L b \mathrm{TDC}$ and MjTDC reveals very different lengths. The structures of other Group II decarboxylases have been published, such as DDC from pig (1JS3, 2.25 $)$, HDC (4E1O, 1.8 $)$ and GAD (2OKJ, $2.3 \AA$ ) from humans. Although they exhibit relatively low amino acid sequence identities $(<30 \%)$ with $L b T D C$, their spatial structures were relatively conserved. All of them consist of three characterized domains and have similar active centers and conserved essential residues, such as the tyrosine in the flexible loop, which is vital for protonation.

In the electron density map, some of the amino acids in the loop region (from 415 to 431) are invisible, and could not be resolved due to substantial disorder. This flexible loop region is located near the substrate-binding site, and is conserved in other Group II decarboxylases. It has been shown that this loop might act as a solvent and substrate/product lid ${ }^{32}$. The conserved tyrosine residue (Y420) in the loop region is responsible for protonating $\mathrm{C}_{\alpha}$ in the transition state ${ }^{32-35}$. Y420A and Y420F mutants were constructed by site-directed mutagenesis. No measureable activity was detected, even in the presence of excess substrate, suggesting the vital role of Y420 in the decarboxylation activity of $L b T D C$.

PLP-binding site. LbTDC is a PLP-dependent enzyme, and the PLP cofactor is covalently attached to the $\varepsilon$-amino group of K392 to form an internal aldimine via a Schiff-base interaction (Fig. 1). The binding between PLP and the enzyme is structurally and functionally conserved in most of the PLP-dependent enzymes. The protonated pyridine nitrogen of PLP forms a pair of salt bridges to the carboxyl group of D328. Moreover, the PLP pyridine ring is anchored by the methyl group of A330 and the imidazole ring of $\mathrm{H} 241$. The O3 atoms of the pyridine ring of PLP seem to interact with T298, K392 and two adjacent water molecules. The phosphate moiety of PLP is stabilized by a number of interactions with G158, S159, and D389 of one subunit and S440 of the other subunit.

The role of $\mathrm{H} 241$. A comparison of the structure of apo- $L b \mathrm{TDC}$ with the structure of holo- $L b \mathrm{TDC}$ revealed that there are two opposite conformations of K240 and H241 (Fig. 1). In the apo-LbTDC structure, the imidazole ring of $\mathrm{H} 241$ is anchored by two water molecules and is distant from the PLP binding pocket, and the side chain of K240 is placed in a position near the PLP binding pocket. However, in the holo- $L b$ TDC structure, the positions of K240 and H241 are rotated to the opposite orientation. Residues K240 and H241 form a short flexible loop. In chain B of holo- $L b T D C$, a conformational shift of this short flexible loop was captured, in contrast to the apo- $L b$ TDC structure. The imidazole ring of $\mathrm{H} 241$ swings to the pyridine ring of PLP. Additionally, through a structural alignment with drDDC and pig DDC, a conserved histidine (H192) was also identified, which has been reported to interact with the pyridine ring of PLP. This interesting dynamic change in conformation suggests that K240 and H241 play important roles in establishing the conformation required for the binding and stabilization of cofactor and substrate.

To investigate the role of K240 and $\mathrm{H} 241$ in $L b T D C$, semi-saturation mutagenesis of $\mathrm{H} 241(\mathrm{H} 241 \mathrm{~A}, \mathrm{H} 241 \mathrm{~N}$, H241Q, H241W, H241R, H241D, and H241F) was performed, and the K240A mutant was created (Fig. 2). All variants were purified for the activity and kinetic analyses, and the mutations had no significant effect on the expression levels and solubility of $L b$ TDC. Compared with the wild type (WT) protein, K240A exhibits an increased $k_{\text {cat }}$ toward tyrosine and a decreased $k_{\text {cat }}$ toward DOPA. Additionally, higher $K_{\mathrm{m}}$ values were observed for both tyrosine and DOPA using the K240A mutant (Table 2). As a result, the $k_{\text {cat }} / K_{\mathrm{m}}$ of K240A toward both tyrosine and DOPA was also decreased, indicating that mutation of lysine-240 to alanine might affect the affinity of enzyme and substrates by altering the conformation of adjacent amino acids, particularly H241. Most of the $\mathrm{H} 241$ mutants completely lost their decarboxylase activity, but $\mathrm{H} 241 \mathrm{~N}$ and $\mathrm{H} 241 \mathrm{Q}$ retained low activities toward tyrosine and DOPA. We failed to determine the kinetic parameters of $\mathrm{H} 241 \mathrm{~N}$ and $\mathrm{H} 241 \mathrm{Q}$, because their $K_{\mathrm{m}}$ values were too high to be determined within the solubility of tyrosine $(5.5 \mathrm{mM})$. Nevertheless, it can still be concluded that the substrate affinity of the H241 variants is significantly decreased, based on the $K_{\mathrm{m}}$ of the WT enzyme toward tyrosine $(0.58 \mathrm{mM})$. We supposed that the mutations of $\mathrm{K} 240$ and $\mathrm{H} 241$ influenced the apo-holo transition and led to weak PLP binding and the loss of decarboxylase activity. K240 and H241 are key residues required for the stabilization of substrate and cofactor. A sequence alignment of $L b T D C$ and other Group II decarboxylase reveals that His 241 is highly conserved ${ }^{36}$. In the crystal structures of drDDC and pig DDC, the corresponding histidine (H192 in drDDC and pig DDC) interacts with the PLP pyridine ring and the inhibitor (cabiDOPA), respectively ${ }^{32,37}$. The residues corresponding to position 240 in most Group II decarboxylases are mainly alanine, 
instead of the lysine observed in $L b$ TDC. In this study, the K240A mutant displayed a decreased $k_{\text {cat }} / K_{\mathrm{m}}$, suggesting a beneficial role of K240 in $L b T D C$.

Site-directed mutagenesis around the active site. Despite the various conditions used to co-crystallize $L b T D C$ with tyrosine or inhibitors (tyrosine methyl ester and tyrosine amide), crystals of the complex with the substrate or inhibitors were not obtained. To further investigate the role of the residues around the catalytic center, tyrosine was docked into the holo- $L b$ TDC structure. Due to its critical role in Parkinson disease, a number of site-directed mutagenesis studies have been conducted on DDC to understand its catalytic mechanism. The K303A mutant showed a 1,500-fold decrease in decarboxylation activity. The kinetic analysis reveals that Lys303 plays an irreplaceable role in the formation of the external aldimine, hydrolysis and product release ${ }^{38}$. The increased $K_{\mathrm{m}}$ values of the T82A and T82S mutants suggest that T82 is involved in substrate binding ${ }^{36}$. Mutation of residues D271, H192, H302, and N300 shows that these residues are not essential but can alter the nature of catalysis $^{39}$. A number of residues within the loops around the putative substrate binding pocket (as illustrated in Fig. $3 \mathrm{~A}$ ) of $L b T D C$ were selected for the site-directed mutagenesis study. All of the residues were mutated to alanine (except for A295F and V296F) because it displays no polar character and less steric hindrance. All variants were purified, and the specific activities were analyzed (Fig. 3B).

Our results show that mutagenesis of the residues between 98 and 103, which are located in a loop region, led to decreased decarboxylase activity. The H98A and S101A variants retained approximately $40 \%$ and $80 \%$ of their activity, respectively, whereas the other mutants were almost completely inactive. Although mutations of residues 294-299 also resulted in decreased activity, a different substrate preference was noticed in variants, such as G296F, which displayed higher activity toward DOPA than tyrosine compared with WT. Therefore, residues 294-299 are considered important for substrate specificity. Future studies could analyze this region to obtain TDC variants with decarboxylase activity toward different amino acids.

Residues 395-398, which are close to the substrate, and Y331 and H391 were also mutated to alanine. H391A and P397A showed approximately $40 \%$ and $50 \%$ of the activity of the WT, respectively, whereas the others displayed extremely low activities $(<5 \%)$. No obvious change in substrate preference was observed in the variants. Further saturation mutagenesis would be necessary to gain deeper insights into the role of this loop. The specific activity of the Y398A mutant was significantly decreased toward both tyrosine and DOPA. Superposition of the pig DDC structure onto the $L b$ TDC structure reveals that the Tyr398 position in $L b$ TDC is a phenylalanine in pig DDC (Fig. 4). Hence, Y398F and Y398A mutants were further characterized to clarify the role of Y398. The $K_{\mathrm{m}}$ values of the Y398A variant toward tyrosine and DOPA are 5- and 2-fold higher than that of the WT enzyme, respectively. For the $\mathrm{Y} 398 \mathrm{~F}$ variant, the $K_{\mathrm{m}}$ toward tyrosine is increased more than 4 -fold, whereas its $K_{\mathrm{m}}$ toward DOPA is slightly reduced by $13 \%$. The $k_{\text {cat }}$ values of Y398F and Y398A toward both substrates are significantly decreased (Table 2). The results indicate that Y398 is important for the binding to the amino acid substrate.

The M505A, S586A, and M588A variants were also constructed and purified. Interestingly, S586A displayed an over 2 -fold improvement in activity compared with the WT enzyme $\left(96.0 \pm 2.0 \mathrm{U} \cdot \mathrm{mg}^{-1}\right.$ versus $43.1 \pm 1.0$ $\left.\mathrm{U} \cdot \mathrm{mg}^{-1}\right)$, representing the highest activity among all variants tested. The kinetic parameters of S586A were also determined. The $k_{\text {cat }}$ and $K_{\mathrm{m}}$ values of S586A toward tyrosine and DOPA are $250.9 \pm 4.2 \mathrm{~s}^{-1}$ and $0.42 \pm 0.03 \mathrm{mM}$ and $126 \pm 3.48 \mathrm{~s}^{-1}$ and $0.69 \pm 0.07 \mathrm{mM}$, respectively. Its $k_{\text {cat }} / K_{\mathrm{m}}$ values toward tyrosine and DOPA are 600 and $181 \mathrm{~s}^{-1} \cdot \mathrm{mM}^{-1}$, respectively, which are 2.78 - and 1.79-fold higher than the WT enzyme. A sequence alignment with other Group II decarboxylases indicates that the residues at 586 are not conserved. Considering the beneficial effect of the alanine mutation at S586, saturation mutagenesis was performed to elucidate its specific mechanisms. In addition to S586A, S586G and S586T retained more than 10\% activity toward tyrosine, whereas the other mutants showed less than $5 \%$ or undetectable activity toward tyrosine or DOPA (Fig. 5). These results suggest that S586 is a critical residue for substrate binding. Based on the activities of S586A, S586G, and S586T compared with WT, it is speculated that there are specific requirements regarding the steric hindrance and hydrophobicity of residue 586. Only amino acids with small or similar side chains to serine at position 586 retained decarboxylase activity, suggesting that reduced steric hindrance is advantageous. Additionally, the docking result indicates that the distance between S586 and tyrosine is only $2.9 \AA$ (Fig. 6). Importantly, hydrophobicity is also attributed to the higher hydropathy index of alanine (2.5), which is higher than glycine $(-0.4)$, serine $(-0.8)$, and threonine $(-0.7)^{40}$, and variant S586A displayed the highest decarboxylase activity. The higher hydrophobicity at position 586 might conduce to enhanced binding energy for tyrosine.

In summary, we determined the crystal structure of $L b T D C$ in complex with PLP at $1.9 \AA$. Semi-saturation mutagenesis on the conformationally flexible $\mathrm{H} 241$ residue shows that only $\mathrm{H} 241 \mathrm{~N}$ and $\mathrm{H} 241 \mathrm{Q}$ retained low activity, indicating that $\mathrm{H} 241$ is vital for the conformation required to bind PLP and tyrosine. Alanine scanning mutagenesis revealed several residues that influence activity and specificity, including G296, Y398, and S586. Saturation mutagenesis was performed on S586. The $k_{\text {cat }} / K_{\mathrm{m}}$ values of the S586A variant toward tyrosine and DOPA are 600 and $181 \mathrm{~s}^{-1} \cdot \mathrm{mM}^{-1}$, which are 2.78- and 1.79-fold higher than the WT enzyme. Decreased steric hindrance and increased hydrophobicity are possible reasons for the improved catalytic efficiency of S586A. This study provided a structural basis for the further engineering and application of TDC in the biocatalytic synthesis of tyramine.

\section{References}

1. Roeder, T., Seifert, M., Kahler, C. \& Gewecke, M. Tyramine and octopamine: Antagonistic modulators of behavior and metabolism. Arch. Insect Biochem. Physiol. 54, 1-13 (2003).

2. Santos, M. H. S. Biogenic amines: Their importance in foods. Int. J. Food Microbiol. 29, 213-231 (1996).

3. Zhu, Z. T., Munhall, A. C. \& Johnson, S. W. Tyramine excites rat subthalamic neurons in vitro by a dopamine-dependent mechanism. Neuropharmacology 52, 1169-1178 (2007). 
4. Molaei, G., Paluzzi, J. P., Bendena, W. G. \& Lange, A. B. Isolation, cloning, and tissue expression of a putative octopamine/tyramine receptor from locust visceral muscle tissues. Arch. Insect. Biochem. Physiol. 59, 132-149 (2005).

5. Lee, K., Kang, K., Park, M., Park, S. \& Back, K. Enhanced octopamine synthesis through the ectopic expression of tyrosine decarboxylase in rice plants. Plant Science 176, 46-50 (2009).

6. Coton, M., Coton, E., Lucas, P. \& Lonvaud, A. Identification of the gene encoding a putative tyrosine decarboxylase of Carnobacterium divergens 508. Development of molecular tools for the detection of tyramine-producing bacteria. Food Microbiol. 21, 125-130 (2004).

7. Zorzano, O. A. C. et al. University of Barcelona. Combination of amines and vanadium (II/IV) compounds for the treatment and prevention of diabetes mellitus. European Patent EP1338280. 2003 August 27.

8. Jie, W. U., Xu, G. U., Dong, L. I., Ai-zhong, D. \& Xiao-yang, D. A. I. Improved synthesis of hypolipidemic drug bezafibrate. Chin. J. New Drugs 19, 311-312 (2010).

9. Sandmeier, E., Hale, T. I. \& Christen, P. Multiple evolutionary origin of pyridoxal-5'-phosphate-dependent amino-acid decarboxylases. Eur. J. Biochem. 221, 997-1002 (1994).

10. Zhang, K. \& Ni, Y. Tyrosine decarboxylase from Lactobacillus brevis: soluble expression and characterization. Protein Expres. Purif. 94, 33-39 (2014).

11. Moreno-Arribas, V. \& Lonvaud-Funel, A. Tyrosine decarboxylase activity of Lactobacillus brevis IOEB 9809 isolated from wine and L. brevis ATCC 367. FEMS Microbiol. Lett. 180, 55-60 (1999).

12. Lehmann, T. \& Pollmann, S. Gene expression and characterization of a stress-induced tyrosine decarboxylase from Arabidopsis thaliana. FEBS Lett. 583, 1895-1900 (2009).

13. Facchini, P. J. \& Deluca, V. Expression in Escherichia coli and partial characterization of two tyrosine-dopa decarboxylases from opium poppy. Phytochemistry 38, 1119-1126 (1995).

14. Cole, S. H. et al. Two functional but noncomplementing Drosophila tyrosine decarboxylase genes: distinct roles for neural tyramine and octopamine in female fertility. J. Biol. Chem. 280, 14948-14955 (2005).

15. Ishida, Y. \& Ozaki, M. Aversive odorant causing appetite decrease downregulates tyrosine decarboxylase gene expression in the olfactory receptor neuron of the blowfly, Phormia regina. Die Naturwissenschaften 99, 71-75 (2012).

16. Moreno-Arribas, V. \& Lonvaud-Funel, A. Purification and characterization of tyrosine decarboxylase of Lactobacillus brevis IOEB 9809 isolated from wine. Fems Microbiology Letters 195, 103-107 (2001).

17. Borresen, T., Klausen, N. K., Larsen, L. M. \& Sorensen, H. Purification and characterisation of tyrosine decarboxylase and aromaticL-amino-acid decarboxylase. Biochim. Et Biophy. Acta 993, 108-115 (1989).

18. Kezmarsky, N. D., Xu, H., Graham, D. E. \& White, R. H. Identification and characterization of a L-tyrosine decarboxylase in Methanocaldococcus jannaschii. Biochim. Et Biophy. Acta 1722, 175-182 (2005).

19. Coton, M. et al. Characterization of the tyramine-producing pathway in Sporolactobacillus sp. P3J. Microbiology 157, 1841-1849 (2011).

20. Thakur, M. \& Azmi, W. Biotransformation of L-tyrosine to tyramine by the growing cells of Lactococcus lactis. Acta Microbiol. Imm. H. 56, 101-114 (2009).

21. Pereira, C. I., Matos, D., Romao, M. V. S. \& Crespo, M. T. B. Dual role for the tyrosine decarboxylation pathway in Enterococcus faecium E17: response to an acid challenge and generation of a proton motive force. Appl. Environ. Microbiol. 75, 345-352 (2009).

22. Doublie, S. Preparation of selenomethionyl proteins for phase determination. Methods Enzymol. 276, 523-530 (1997).

23. Otwinowski, Z. \& Minor, W. Processing of X-ray diffraction data collected in oscillation mode. Methods Enzymol. 276, 307-326 (1997).

24. Terwilliger, T. C. et al. Decision-making in structure solution using Bayesian estimates of map quality: the PHENIX AutoSol wizard. Acta Crystallogr. Sect. D-Biol. Crystallogr. 65, 582-601 (2009).

25. Terwilliger, T. C. et al. Iterative model building, structure refinement and density modification with the PHENIX AutoBuild wizard. Acta Crystallogr. Sect. D-Biol. Crystallogr. 64, 61-69 (2008).

26. Adams, P. D. et al. PHENIX: a comprehensive Python-based system for macromolecular structure solution. Acta Crystallogr. Sect. D-Biol. Crystallogr. 66, 213-221 (2010).

27. Emsley, P., Lohkamp, B., Scott, W. G. \& Cowtan, K. Features and development of Coot. Acta Crystallogr. Sect. D-Biol. Crystallogr. 66, 486-501 (2010).

28. McCoy, A. J. et al. Phaser crystallographic software. J. Appl. Crystallogr. 40, 658-674 (2007).

29. Potterton, L. et al. Developments in the CCP4 molecular-graphics project. Acta Crystallogr. Sect. D-Biol. Crystallogr. 60, 2288-2294 (2004).

30. G. Giardina. et al. Open conformation of human DOPA decarboxylase reveals the mechanism of PLP addition to Group II decarboxylases. Proc. Natl. Acad. Sci. USA 108, 20514-20519 (2011).

31. Schneider, G., Kack, H. \& Lindqvist, Y. The manifold of vitamin B-6 dependent enzymes. Structure with Folding \& Design 8, R1-R6 (2000).

32. Burkhard, P., Dominici, P., Borri-Voltattorni, C., Jansonius, J. N. \& Malashkevich, V. N. Structural insight into Parkinson's disease treatment from drug-inhibited DOPA decarboxylase. Nature Struc. Biol. 8, 963-967 (2001).

33. Fenalti, G. et al. GABA production by glutamic acid decarboxylase is regulated by a dynamic catalytic loop. Nature Struc. Mol. Biol. 14, 280-286 (2007)

34. Bertoldi, M., Gonsalvi, M., Contestabile, R. \& Voltattorni, C. B. Mutation of tyrosine 332 to phenylalanine converts dopa decarboxylase into a decarboxylation-dependent oxidative deaminase. J. Biol. Chem. 277, 36357-36362 (2002).

35. Komori, H., Nitta, Y., Ueno, H. \& Higuchi, Y. Structural study reveals that Ser-354 determines substrate specificity on human histidine decarboxylase. J. Biol. Chem. 287, 29175-29183 (2012).

36. Ishii, S., Mizuguchi, H., Nishino, J., Hayashi, H. \& Kagamiyama, H. Functionally important residues of aromatic L-amino acid decarboxylase probed by sequence alignment and site-directed mutagenesis. J. Biochem. 120, 369-376 (1996).

37. Han, Q., Ding, H., Robinson, H., Christensen, B. M. \& Li, J. Crystal structure and substrate specificity of Drosophila 3,4dihydroxyphenylalanine decarboxylase. PloS ONE 5, e8826 (2010).

38. Bertoldi, M. \& Voltattorni, C. B. Multiple roles of the active site lysine of Dopa decarboxylase. Arch. Biochem. Biophys. 488, 130-139 (2009).

39. Bertoldi, M., Castellani, S. \& Voltattorni, C., B. Mutation of residues in the coenzyme binding pocket of Dopa decarboxylase. Eur. J. Biochem. 268, 2975-2981 (2001).

40. Kyte, J. \& Doolittle, R. F. A simple method for displaying the hydropathic character of a protein. J. Mol. Biol. 157, 105-132 (1982).

\section{Acknowledgements}

We are grateful to the National Natural Science Foundation of China (21276112 and 21506073), a Grant from Science and Technology Commission of Shanghai Municipality (15JC1400403), the Natural Science Foundation of Jiangsu Province (BK20150003), the Fundamental Research Funds for the Central Universities (JUSRP51409B), the Program of Introducing Talents of Discipline to Universities (111-2-06), and a project 
funded by the Priority Academic Program Development of Jiangsu Higher Education Institutions for the financial support of this research.

\section{Author Contributions}

H.Z. performed the protein expression, purification and crystallization; data collection, mutagenesis, activity and kinetic assays and molecular docking experiments; analyzed the data; and drafted the manuscript. G.X. assisted in designing the experiments and revised the manuscript. K.Z. performed the crystallization experiments and analyzed the data. X.K. provided technical assistance in protein crystallization. R.H. revised the manuscript. J.Z. designed the crystallization experiments and revised the manuscript. Y.N. designed the study and revised the manuscript. All authors read and approved the final manuscript.

\section{Additional Information}

Supplementary information accompanies this paper at http://www.nature.com/srep

Competing financial interests: The authors declare no competing financial interests.

How to cite this article: Zhu, H. et al. Crystal structure of tyrosine decarboxylase and identification of key residues involved in conformational swing and substrate binding. Sci. Rep. 6, 27779; doi: 10.1038/srep27779 (2016).

(c) (1) This work is licensed under a Creative Commons Attribution 4.0 International License. The images or other third party material in this article are included in the article's Creative Commons license, unless indicated otherwise in the credit line; if the material is not included under the Creative Commons license, users will need to obtain permission from the license holder to reproduce the material. To view a copy of this license, visit http://creativecommons.org/licenses/by/4.0/ 